\title{
Moderate Hypertriglyceridemia Revealed by Acute Chest Syndrome, a Milky Appearance Serum and Prior History of Recurrent Acute Pancreatitis in a Type 2 Diabetes Black Patient: A Case Report
}

\author{
François Bompeka Lepira ${ }^{1}$, Vita Eulethère Kintoki², Jean-Robert Risassi Makulo1, \\ Fabien Mbala Kintoki² \\ ${ }^{1}$ Division of Nephrology-Hypertension, University of Kinshasa Hospital, Kinshasa, Democratic Republic of the Congo \\ ${ }^{2}$ Division of Cardiology, University of Kinshasa Hospital, Kinshasa, Democratic Republic of the Congo \\ Email: ${ }^{*}$ lepslepira@yahoo.fr
}

How to cite this paper: Lepira, F.B., Kintoki, V.E., Makulo, J.R. and Kintoki, F.M. (2016) Moderate Hypertriglyceridemia Revealed by Acute Chest Syndrome, a Milky Appearance Serum and Prior History of Recurrent Acute Pancreatitis in a Type 2 Diabetes Black Patient: A Case Report. Worla Journal of Cardiovascular Diseases, 6, 425432.

http://dx.doi.org/10.4236/wjcd.2016.611046

Received: August 30, 2016

Accepted: November 27, 2016

Published: November 30, 2016

Copyright $\odot 2016$ by authors and Scientific Research Publishing Inc. This work is licensed under the Creative Commons Attribution International License (CC BY 4.0).

http://creativecommons.org/licenses/by/4.0/

\section{Abstract}

Although the "triglyceride paradox" states that hypertriglyceridemia is less frequent in Blacks and the risk of pancreatitis increases with severe hypertriglyceridemia, we herein report on a case of moderate hypertriglyceridemia revealed by an acute chest syndrome and a milky appearance serum in a 47-year-old type 2 diabetes black patient with prior history of recurrent acute pancreatitis. In addition to insulin therapy and coronary angioplasty, the combination of a statin and a fibrate resulted two months later in a substantial improvement in triglyceride levels and a normal serum appearance.

\section{Keywords}

Moderate Hypertriglyceridemia, Acute Chest Syndrome, Pancreatitis, Fibrates, Black African

\section{Introduction}

Dyslipidemia in T2DM is characterized by an atherogenic profile with elevated lowdensity lipoprotein-cholesterol (LDL-c), elevated triglycerides (TG) and decreased highdensity lipoprotein-cholesterol (HDL-c) and increases the risk of both coronary artery disease (CAD) [1] and pancreatitis [2]. Indeed, modest increase in TG levels has been reported to be associated with CVD risk whereas severe hypertriglyceridemia (TG > $11.3 \mathrm{mmol} / \mathrm{l}$ ) increases the risk of pancreatitis [1] [3]. If the role of HTG in promoting 
pancreatitis is well accepted, controversy still remains about CVD [3]. However, the clustering of HTG with other components of the metabolic syndrome such as elevated small dense LDL-c particles and low HDL-c could explain the increased CVD risk conferred by HTG [4]. Although the so-called "triglyceride paradox" states that hypertriglyceridemia (HTG) is less frequent in blacks [5] [6], we herein report on a case of moderate HTG revealed by an acute chest syndrome and a milky appearance serum in a 47 years old poorly controlled T2DM black patient with prior history of recurrent acute pancreatitis referred to the outpatient clinic of the Division of Cardiology of the University of Kinshasa Hospital.

\section{Case Report}

IB is a 47 years old black patient with type 2 diabetes under mixed insulin since 1994. In August 2014, due to an acute chest pain on walking that usually relieves 3 to 4 minutes after stopping walking, his general practitioner from Brazzaville, Republic of Congo referred him to the outpatient clinic of the Division of Cardiology/University of Kinshasa Hospital for, Republic of Congo. His past medical history is characterized by a history of recurrent acute pancreatitis (2002 and 2003) and hypertension (2009) successfully controlled with a combination of angiotensin converting enzyme inhibitor (Ramipril 10 $\mathrm{mg}$ ) and thiazide diuretic (Hydrochlorothiazide $12.5 \mathrm{mg}$ ). He was not smoking or consuming alcohol and has no physical activity in leisure time. Physical examination revealed a body mass index (BMI), waist circumference (WC), seated blood pressure (BP), pulse rate of $29 \mathrm{Kg} / \mathrm{m}^{2}, 98 \mathrm{~cm}, 142 / 89 \mathrm{~mm} \mathrm{Hg}$ and $98 \mathrm{bpm}$, respectively (Table 1). Cardiac and pulmonary sounds were normal; there was no abdominal tenderness, hepatomegaly nor splenomegaly; pedal and posterior arterial pulses were normally perceived and symmetric. Cardiac ultrasound was normal and electrocardiogram findings of negative $\mathrm{T}$ waves at leads $\mathrm{D} 1, \mathrm{aVL}, \mathrm{V} 1, \mathrm{~V} 4, \mathrm{~V} 5$ and V6 were suggestive of inferior and lateral ischemia. The diagnosis of acute chest syndrome complicating a metabolic syndrome (MetS) was retained and patient was prescribed, in addition to current antihypertensive treatment, an oral cardio-selective beta-blocker (Atenolol $10 \mathrm{mg}$ ), nitrate compound (Isosorbide dinitrate $10 \mathrm{mg}$ ), statin (Simvastatin $10 \mathrm{mg}$ ) and antiplatelet (Clodopigrel $75 \mathrm{mg}$ ) with progressive relieve of chest pain. A venipuncture that draw a milky appearance blood sample (Figure 1(a)) was performed and blood chemistry showed fasting plasma glucose (FPG) $21 \mathrm{mmol} / \mathrm{l}$, serum creatinine $159 \mu \mathrm{mol}$ with estimated glomerular filtration rate (eGFR) by abbreviated modification of diet in renal disease equation (MDRD) $52 \mathrm{ml} / \mathrm{min} / 1.73 \mathrm{~m}^{2}$, tri-iodothyroxine (T3) $0.95 \mathrm{ng} / \mathrm{ml}$, tetraiodothyroxine (T4) $103 \mathrm{nmol} / \mathrm{l}$ and thyroid stimulating hormone (TSH) $0.8 \mathrm{IU} / \mathrm{ml}$. Lipid and lipoprotein profile was as follows: total cholesterol (TC) $9.87 \mathrm{mmol} / \mathrm{l}$, LDL-c $5.14 \mathrm{mmol} / \mathrm{l}$, HDL-c $1.0 \mathrm{mmol} / \mathrm{l}$, non-HDL-c $8.86 \mathrm{mmol} / \mathrm{l}$, TG $8.09 \mathrm{mmol} / \mathrm{l}$ (Table 2). The analysis of these laboratory tests revealed a mixed dyslipidemia (elevated TG and LDL-c) with low HDL-c and a reduced kidney function. Abdominal ultrasound showed pancreatic micro-calcifications suggestive of asymptomatic chronic pancreatitis. Finally, the diagnosis of acute coronary syndrome (ACS) complicating a T2DM-associated 
Table 1. Sociodemographic and clinical characteristics of the patient at the first visit.

\begin{tabular}{|c|c|}
\hline Variable & \\
\hline Age, years & 47 \\
\hline \multicolumn{2}{|l|}{ Past medical history: } \\
\hline \multicolumn{2}{|l|}{-Diabetes (1994) } \\
\hline \multicolumn{2}{|l|}{-Acute pancreatitis $(2002,2009)$} \\
\hline \multicolumn{2}{|l|}{-Hypertension (2009) } \\
\hline $\mathrm{BMI}, \mathrm{Kg} / \mathrm{m}^{2}$ & 29 \\
\hline Waist circumference, $\mathrm{cm}$ & 98 \\
\hline SBP, mm Hg & 149 \\
\hline DBP, mm Hg & 89 \\
\hline Pulse rate, bpm & 98 \\
\hline $\mathrm{FPG}, \mathrm{mmol} / \mathrm{l}$ & 21 \\
\hline Serum creatinine, $\mu \mathrm{mol} / 1$ & 159 \\
\hline GFR-MDRD, $\mathrm{ml} / \mathrm{min} / 1.73 \mathrm{~m}^{2}$ & 52 \\
\hline Triiodothyroxin (T3), ng/ml & 0.95 \\
\hline Tetraiodothyroxin (T4), nmol/l & 106 \\
\hline FSH, IU/l & 0.80 \\
\hline
\end{tabular}

Abbreviations: BMI, body mass index; SBP, systolic blood pressure; DBP, diastolic blood pressure; FPG, fasting plasma glucose; GFR, glomerular filtration rate; MDRD, modification of diet in renal disease; Follicle stimulating hormone; IU, international unit.

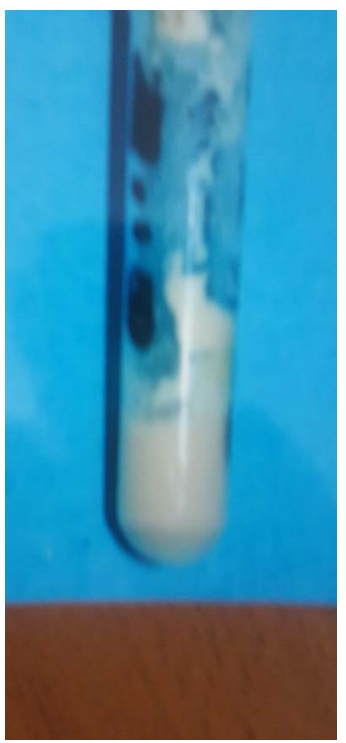

(a)

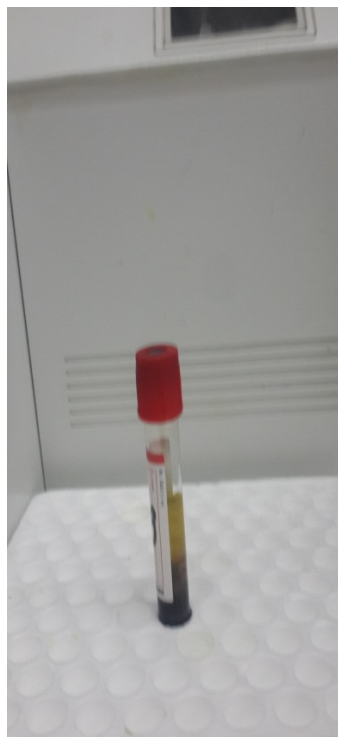

(b)

Figure 1. Serum appearance before (a) and after (b) two months treatment with the combination of statin and fibrate (Fenofibrate). 
Table 2. Lipid and lipoprotein profile before and two months after the addition of a fibrate to the statin.

\begin{tabular}{ccc}
\hline Variable & Before fibrate & After fibrate \\
\hline Total cholesterol mmol/l & 9.87 & 6.80 \\
LDL-c, mmol/l & 5.14 & 3.37 \\
HDL-c, mmol/l & 1.00 & 1.26 \\
Non HDL-c, mmol/l & 8.86 & 5.29 \\
TG, mmol/l & 8.09 & 4.86 \\
\hline
\end{tabular}

Abbreviations: LDL-c, low-density lipoprotein-cholesterol; HDL-c, high-density lipoprotein-cholesterol; TG, triglycerides.

mixed dyslipidemia with mainly HTG as a component of MetS was retained. Thiazide diuretic and beta-blocker, drugs known to alter glucose and lipid metabolism, were stopped and replaced by oral low release indapamide $(2.5 \mathrm{mg})$ and an angiotensin converting enzyme inhibitor Ramipril (10 mg), respectively. Since statins do not effectively lower TG levels, a fibrate (Oral Fenofibrate $160 \mathrm{mg}$ once daily) was added to statin. Two months later (November 2014), the patient was feeling better and has no complaints. Venipuncture drawn a red blood sample (Figure 1(b)) and laboratory parameters were as follows: FPG $8 \mathrm{mg} / \mathrm{dl}$, blood urea nitrogen (BUN) $6.8 \mathrm{mmol} / \mathrm{l}$, creatinine $124 \mu \mathrm{mol} / \mathrm{l}$, eGFR-MDRD $69.8 \mathrm{ml} / \mathrm{min} / 1.73 \mathrm{~m}^{2}$, TC $6.56 \mathrm{mmol} / \mathrm{l}$, LDL-c $3.97 \mathrm{mmol} / \mathrm{l}$, HDL-c $1.26 \mathrm{mmol} / \mathrm{l}$, non-HDL-c $5.29 \mathrm{mmol} / \mathrm{l}$, TG $4.8 .6 \mathrm{mmol} / \mathrm{l}$ (Table 2). As coronary angiography was not available in Kinshasa, the patient travelled abroad to Lazio in Italy where coronary angiography evidenced an occlusion of descendant anterior coronary artery and a stenosis of circumflex artery. Coronary angioplasty with stent was performed on January 10, 2015 and patient was prescribed oral cardioaspirin $100 \mathrm{mg}$, Lopressor $100 \mathrm{mg}$, Ramipril $10 \mathrm{mg}$, Eskim $1000 \mathrm{mg}$, Atorvastatin $40 \mathrm{mg}$, Pantoprazole 40 $\mathrm{mg}$ and subcutaneous Novorapid $18 \mathrm{IU}$ thrice daily and Levemir $18 \mathrm{IU}$ once daily. At discharge, the patient was feeling better and went back to Brazzaville under the surveillance of his general practitioner.

\section{Discussion}

We reported on a 47 -year-old patient with type 2 diabetes with prior history of acute pancreatitis presenting with an acute chest pain and very important hypertriglyceridemia with a milky appearance serum.

\subsection{Clinical Findings}

Lipid profile made of increased levels of both cholesterol and TG observed in the present T2DM diabetes case is a well-known feature of mixed dyslipidemia and does translate underlying insulin resistance state [1]. Indeed, insulin resistance has been reported to be associated with an increase in serum TG through several mechanisms including reduced insulin-dependent inhibition of lipolysis in adipocytes, increased TG 
and very-low lipoprotein density cholesterol (VLDL-c) production by the liver, and impaired insulin-dependent activation of lipoprotein lipase and hydrolysis of TG-rich lipoprotein particles with subsequent fasting and postprandial HTG [7]. In the absence of genetic testing, contributing factors to HTG in the present case could be physical inactivity, central obesity, uncontrolled hypertension and diabetes, reduced kidney function, and the use of drugs known to alter lipid metabolism such as thiazide diuretic and beta blocker; diet could be another factor but it was not formally evaluated in the present case [7].

A milky appearance of serum in the present case does suggest high levels of chylomicrons as observed in type V hyperlipoproteinemia [8]. Chylomicronemia syndrome defined as TG levels above $11.3 \mathrm{mmol} / \mathrm{l}$ plus one of either eruptive xanthoma, lipemia retinalis or abdominal pain/pancreatitis is a complication of HTG usually considered as rare [8]. However, a serum milky in color can be seen with TG levels between -11.3 $\mathrm{mmol} / \mathrm{l}$ since chylomicrons are formed at TG levels $>5.65 \mathrm{mmol} / \mathrm{l}$ [9]. In this regard, Zhang et al. [9] in a retrospective clinical study of 224 patients with hypertriglyceridemia pancreatitis that TG $\geq 5.65 \mathrm{mmol} / \mathrm{l}$ should raise a high degree of suspicion of HTG, especially if no other etiology of acute pancreatitis is apparent.

Patient's past-medical history was characterized by two episodes of acute pancreatitis. Hypertriglyceridemia is a well-established cause of acute pancreatitis, accounting for $1 \%-4 \%$ to $9 \%$ of patients who present with acute pancreatitis [10] [11] [12] [13]. Although the exact pathway by which HTG causes acute pancreatitis remains poorly understood, two potential mechanisms have been suggested [9] [14]. The first one relies on the hydrolysis of excess TG by pancreatic lipase causing accumulation of free fatty acids in the pancreas; the latter in turn cause acinar cell and pancreatic capillary injury. The resultant ischemia creates an acidic environment, which further enhance free fatty acid toxicity. The second one is that increased chylomicron-induced hyperviscosity in the pancreatic capillaries leads to ischemia [9] [14].

Acute retrosternal chest pain experienced by the patient could be related to the coexistence of multiple CVD risk factors clustering in metabolic syndrome. However, HTG could be one of the most important factors in the development and progression of the dynamic process of atherosclerosis [1]. The Prospective Cardiovascular Munster (PROCAM) study [15] found increases in in risk as TG levels rose from $2.3 \mathrm{mmol} / \mathrm{l}$ to $9.0 \mathrm{mmol} / \mathrm{l}$ after adjustment for other risk factors for cardiovascular disease. A strong independent relation between plasma TG levels and likelihood of cardiovascular disease has been reported in other studies [15]. Meta-analyses of thousands of patients followed up for more than 10 years showed that a TG elevation of $1 \mathrm{mmol} / \mathrm{l}$ increased the risk of cardiovascular disease by $32 \%$ in men and $76 \%$ in women, independent of HDL-c levels [15]. Although the role of HTG in promoting CVD remains still a matter of controversy, recent studies reported a consistent strong positive relationship between TG levels (especially non fasting TG) and CVD [16]. Indeed, HTG has been reported to be associated with low HDL-c and increased levels of the more atherogenic small dense LDL-c particles with subsequent oxidative stress, inflammation and prothrombotic state, all well-known characteristic features of the atherosclerosis process [1] [17]. 


\subsection{Therapeutic Aspects}

\subsubsection{Insulin Therapy}

Despite the presence of metabolic syndrome suggestive of underlying insulin resistance, the present case was paradoxically receiving insulin to control his T2DM. In case of severe T2DM-associated HTG and poorly controlled diabetes as in the present, continuous intravenous insulin infusion has been reported to be beneficial in reducing plasma glucose and serum TG [11]. A recent study, a group of 15 patients with a median TG of $26.23 \mathrm{mmol} / \mathrm{l}$ has their TG levels corrected to a median of $5.75 \mathrm{mmol} / \mathrm{l}$ at discharge with an average $48 \mathrm{~h}$ of continuous insulin infusion [18]. Since TG levels in the present patient were below those reported in aforementioned study, subcutaneous insulin was preferred to continuous intravenous insulin.

\subsubsection{Lipid Lowering Therapy and Hypertriglyceridemia}

Improvement in serum appearance and lipid profile was observed in the present case two months after the initiation of lipid lowering therapy with a statin and a fibrate. Although statins have some TG lowering effects at higher doses, ranging from $20 \%$ to $28 \%$, they are not effective enough to remove the risk of pancreatitis in patients with severe HTG and should not be used in their own as first line agents [2]. In these patients, fibrates are the most efficacious first line pharmacotherapy for HTG, and typically lower TG levels rapidly and effectively [2]. Meta-analyses as well as the ACCORD study suggest that fibrates are useful for the treatment of HTG in diabetic patients [7] [19] [20]. Fenofibrate consistently decreased TG levels to a significantly greater extent than placebo; significantly greater increases in HDL-c levels and significantly greater reductions in LDL-c and total cholesterol levels were also seen in some trials [20]. The beneficial effects of fenofibrate on lipid profile are mediated by mainly the activation of peroxisome proliferator-activated receptor-alpha [21]. Fibrates are generally well tolerated and rarely cause myopathy or hepatic toxicity as showed by safety data from the FIELD study as well the ACCORD lipid trial [14] [21].

\subsubsection{Antihypertensive Therapy}

Beta blocker and thiazide diuretic, drugs known to alter glucose and lipid metabolism, were replaced by an angiotensin converting enzyme inhibitor Ramipril and another diuretic indapamide known to have beneficial or neutral effects on glucose and lipid metabolism. Angiotensin 2 alters insulin secretion and sensitivity through the production of pro-inflammatory cytokines and reactive oxygen species with subsequent oxidative [22]. Thus, the inhibition of renin angiotensin system can improve alterations in glucose and lipid metabolism in diabetes [22].

\section{Conclusion}

A milky serum appearance in the context of acute abdominal pain should guide the doctor to a diagnosis of acute pancreatitis, and to propose the triglyceride assay. We have shown here that the complications of hypertriglyceridemia can be seen in black patients, even at relatively moderate rates of triglycerides. 


\section{Acknowledgements}

The authors would like to gratefully thank all the staff of the Division of Cardiology of the University of Kinshasa Hospital for the administrative authorization. They are deeply indebted to the patient who by his consent allows the caregivers to improve their skills in providing care to T2DM patients with dyslipidemia.

\section{Authors' Contribution}

LFB wrote the manuscript, KEV collected data and revised the manuscript, MJR revised the manuscript, KFM revised the manuscript.

\section{References}

[1] Subramanian, S. and Chait, A. (2012) Hypertriglyceridemia Secondary to Obesity and Diabetes. Biochimica et Biophysica Acta, 1821, 819-825.

http://dx.doi.org/10.1016/j.bbalip.2011.10.003

[2] Sandhu, S., Al-Sarral, A., Taraboanta, C., et al. (2011) Incidence of Pancreatitis, Secondary Causes, and Treatment of Patients Referred to a Special Lipid Clinic with Severe Hypertriglyceridemia: A Retrospective Cohort Study. Lipids in Health and Disease, 10, 157. http://dx.doi.org/10.1186/1476-511X-10-157

[3] Berglund, L., Brunzell, J.D., Goldberg, A.C., et al. (2012) Evaluation and Treatment of Hypertriglyceridemia: An Endocrine Society Clinical Practice Guideline. Journal of Clinical Endocrinology \& Metabolism, 97, 2969-2989. http://dx.doi.org/10.1210/jc.2011-3213

[4] Grundy, S.M. (1999) Hypertriglyceridemia, Insulin Resistance, and the Metabolic Syndrome. American Journal of Cardiology, 83, 25-29.

[5] Yu, S.K., Natalie, L.M., Ramsey, G., et al. (2013) Triglyceride-Based Screening Tests Fail to Recognize Cardiometabolic Disease in African Immigrant and African American Men. Metabolic Syndrome and Related Disorders, 11, 15-20. http://dx.doi.org/10.1089/met.2012.0114

[6] Cook, C., Erdman, D.M., Ryan, G.J., et al. (2014) The Pattern of Dyslipidemia among African-Americans with Type 2 Diabetes. Diabetes Care, 5, 319-324.

[7] Pang, J., Chan, D. and Watts, G. (2014) Origin and Therapy for Hypertriglyceridemia in Type 2 Diabetes. World Journal of Diabetes, 5.

[8] Digby, M., Belli, R., McGraw, T., et al. (2011) Eruptive Xanthomas as a Cutaneous Manifestation of Hypertriglyceridemia. A Case Report. Journal of Clinical and Aesthetic Dermatology, 4, 44-46.

[9] Zhang, X.L., Li, F., Zen, Y.M., Li, A. and Fang, Y. (2015) Clinical Study of 224 Patients with Hypertriglyceridemia Pancreatitis. Chinese Medical Journal, 128, 2045-2049.

[10] Ivanova, R., Puerta, S., Garrido, A., Cueto, I., Ferro, A., Ariza, M.J., et al. (2012) Triglyceride Levels and Apolipoprotein E Polymorphism in Patients with Acute Pancreatitis. Hepatobiliary \& Pancreatic Diseases International, 11, 96-101. http://dx.doi.org/10.1016/S1499-3872(11)60131-8

[11] Denecker, N. and Decochez, K. (2013) Poorly Controlled Type Diabetes Complicated by an Episode of Severe Hypertriglyceridemia-Induced Pancreatitis. BMJ Case Report, Online.

[12] Charlesworth, A., Steger, A. and Crook, M.A. (2015) Acute Pancreatitis with Severe Hypertriglyceridemia. A Retrospective Cohort Study. International Journal of Surgery, 23, 23-27. http://dx.doi.org/10.1016/j.ijsu.2015.08.080 
[13] Carr, R.A., Rejowski, B.J., Cote, G.A., Pitt, H.A. and Zyromski, N.J. (2016) Systematic Review of Hypertriglyceridemia-Induced Acute Pancreatitis: A More Virulent Etiology? Pancreatology, 16, 469-476. http://dx.doi.org/10.1016/j.pan.2016.02.011

[14] Stefanutti, C., Labbadia, G. and Morozzi, C. (2013) Severe Hypertriglyceridemia-Related Acute Pancreatitis. Therapeutic Apheresis and Dialysis, 17, 130-137. http://dx.doi.org/10.1111/1744-9987.12008

[15] Yuan, G., Al-Shali, K.Z. and Hegele, R.A. (2007) Hypertrigyceridemia: Its Etiology, Effects and Treatment. Canadian Medical Association Journal, 176, 1113-1120. http://dx.doi.org/10.1503/cmaj.060963

[16] Lisak, M., Demarin, V., Trkanjec, Z. and Basic-Kes, V. (2012) Hypertriglyceridemia as a Possible Independent Risk Factor for Stroke. Acta Clinica Croatica, 52, 458-463.

[17] Talayero, B.G. and Sacks, F.M. (2011) The Role of Triglycerides in Atherosclerosis. Current Cardiology Reports, 13, 544-552. http://dx.doi.org/10.1007/s11886-011-0220-3

[18] Poonuru, S., Pathak, S.R., Vats, H.S., et al. (2011) Rapid Reduction of Severely Elevated Serum Triglycerides with Insulin Infusion, Gemfibrozil and Niacin. Clinical Medicine Research, 9, 38-41. http://dx.doi.org/10.3121/cmr.2010.898

[19] Tsuang, W., Navaneethan, U., Ruiz, L., et al. (2009) Hypertriglyceridemic Pancreatitis: Presentation and Management. American Journal of Gastroenterology, 104, 984-991. http://dx.doi.org/10.1038/ajg.2009.27

[20] The ACCORD Study Group (2010) Effects of Combination Lipid Therapy in Type 2 Diabetes Mellitus. New England Journal of Medicine, 362, 1563-1574. http://dx.doi.org/10.1056/NEJMoa1001282

[21] Keating, G.M. (2011) Fenofibrate: A Review of Its Lipid-Modifying Effects in Dyslipidemia and Its Vascular Effects in Type 2 Diabetes Mellitus. American Journal of Cardiovascular Drugs, 11, 227-247. http://dx.doi.org/10.2165/11207690-000000000-00000

[22] Favre, G.A., Esnault, V.L. and Van Obberghen, E. (2015) Modulation of Glucose Metabolism by the Renin Angiotensin Aldosterone System. American Journal of Physiology-Endocrinology and Metabolism, 308, E435-E449. http://dx.doi.org/10.1152/ajpendo.00391.2014

Submit or recommend next manuscript to SCIRP and we will provide best service for you:

Accepting pre-submission inquiries through Email, Facebook, LinkedIn, Twitter, etc.

A wide selection of journals (inclusive of 9 subjects, more than 200 journals)

Providing 24-hour high-quality service

User-friendly online submission system

Fair and swift peer-review system

Efficient typesetting and proofreading procedure

Display of the result of downloads and visits, as well as the number of cited articles

Maximum dissemination of your research work

Submit your manuscript at: http://papersubmission.scirp.org/

Or contact wjcd@scirp.org 